\title{
Impact of a Wellness Portal on the Delivery of Patient-Centered Preventive Care
}

\author{
Zsolt Nagykaldi, PhD, Cheryl B. Aspy, PhD, Ann Chou, PhD, MPH, MA, and \\ James W. Mold, MD, MPH
}

Background: The objective of this study was to determine the impact of the Wellness Portal-a novel, web-based patient portal that focuses on wellness, prevention, and longitudinal health-on the delivery of patient-centered preventive care by examining the behavior and experiences of both patients and primary care clinicians and the degree to which recommended services were individualized and provided.

Methods: We conducted a 3-year, systematic portal development and testing study, which included a 6-month feasibility and acceptability pilot in 2 primary care practices followed by a 12-month cluster randomized controlled trial in 8 clinician practices ( 4 in each study group). Descriptive and bivariate analyses were conducted to compare service delivery between intervention and control arms.

Results: Ninety percent of patients in the pilot study found the portal easy to use, $83 \%$ found it to be a valuable resource, and $80 \%$ said that it facilitated their participation in their own care. The cluster randomized controlled trial included 422 adults 40 to 75 years of age and the parents of 116 children 2 to 5 years of age. Seventy three percent of patients used the portal during the study. Both patient activation (measured via the 13-item Patient Activation Measure) and participants' perception of patient-centeredness of care (measured via the Consumer Assessment of Healthcare Providers and Systems instrument) increased significantly in the portal group compared with control $(P=.0014$ and $P=.037$, respectively). A greater proportion of portal users received all recommended preventive services (84.4\% intervention vs $67.6 \%$ control; $P<.0001)$; took low-dose aspirin, if indicated $(78.6 \%$ intervention vs 52.3\% control; $P<.0001)$; and received Pneumovax because of chronic health conditions $(82.5 \%$ vs $53.9 \% ; P<.0001)$ and age $(86.3 \%$ vs $44.6 \% ; P<.0001)$, despite having fewer visits over the study period compared with those in the control group (average of 2.9 vs 4.3 visits; $P<.0001$ ). Children in the intervention group received $95.5 \%$ of all recommended immunizations compared with 87.2\% in the control group $(P=.044)$.

Conclusions: A comprehensive patient portal integrated into the regular process of primary care can increase the patient-centeredness of care, improve patient activation, enhance the delivery of both ageand risk factor-appropriate preventive services, and promote the utilization of web-based personal health records. (J Am Board Fam Med 2012;25:158-167.)

Keywords: Community Medicine, Computers, Evidence-Based Medicine, Medical Decision-Making, Patient-Centered Care, Personal Health Records, Practice-Based Research, Primary Health Care, Prevention, Wellness Portal

As the number of recommended preventive services continues to increase,${ }^{1}$ clinicians struggle to main-

This article was externally peer reviewed.

Submitted 22 April 2011; revised 25 August 2011; accepted 7 September 2011.

From the Department of Family and Preventive Medicine, University of Oklahoma Health Sciences Center, Oklahoma City (ZN, CBA, JWM); and the Veterans Affairs Health Services Research \& Development, Center of Excellence for the Study of Healthcare Provider Behavior, The Greater Los Angeles VA Health Care System, Los Angeles, California (AC). tain a balance between immediate patient concerns and the time required to address prevention., ${ }^{2,3}$ Without effective and timely clinical decision sup-

Funding: Support for this study was provided by the Agency for Healthcare Research and Quality grant no. 1 R18 HS017188-01.

Conflict of interest: none declared.

Corresponding author: Zsolt Nagykaldi, PhD, Department of Family and Preventive Medicine, University of Oklahoma Health Sciences Center, 900 NE 10th Street, Oklahoma City, OK 73104 (E-mail: znagykal@ouhsc.edu). 
Figure 1. Conceptual model of the impact of the Wellness Portal on patient-centered care. PBRN, practice-based research network (primary care); PSRS, preventive services reminder system (a practice-based patient registry and decision support system).

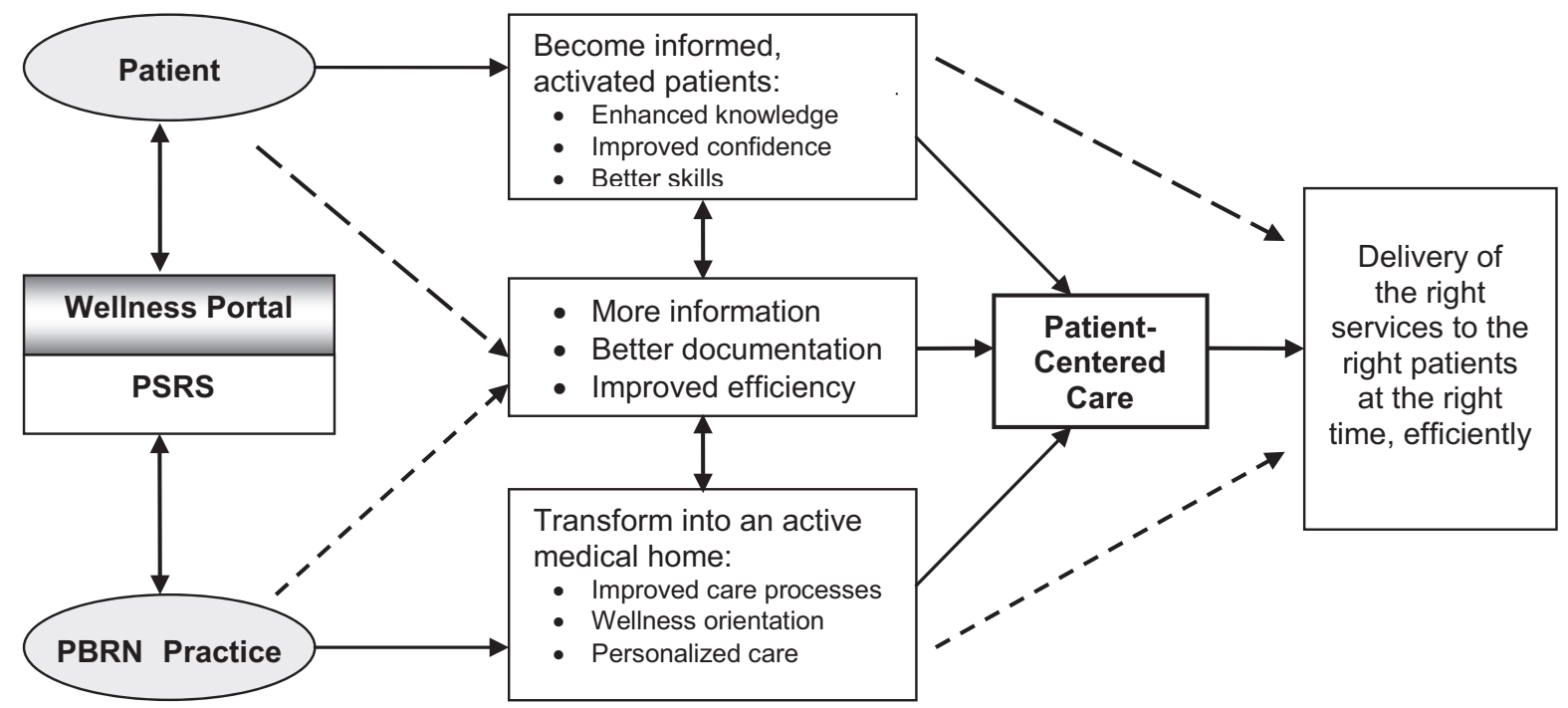

port integrated into a comprehensive care delivery approach (eg, the chronic care model) and without patient-centered tailoring of recommendations, primary care clinicians' performance in this area is likely to go from suboptimal ( $40 \%$ to $60 \%$ rates of delivery of well-accepted preventive services) $)^{4,5}$ to unsatisfactory. Optimal delivery of primary, secondary, and tertiary preventive services increasingly will require sophisticated information processing $^{6}$ and greater patient involvement. ${ }^{7}$ Despite the importance of patient-centered delivery approaches, however, limited information is available about the impact of integrated, patient-driven health information technology on the delivery of patient-centered, preventive care in primary care settings.

Building on our experience with a comprehensive preventive services registry and clinical decision support system we had developed and tested previously, ${ }^{6}$ the Preventive Services Reminder System (PSRS), we conducted a 3-year study to (1) develop, field test, and refine a web-based patient Wellness Portal linked to PSRS to facilitate patient-centered, preventive care in primary care practices; and (2) determine the impact of the Wellness Portal on the process of patient-centered preventive care by examining the behavior and experiences of both patients and providers and the degree to which recommended services were individualized and delivered. We expected that acti- vated patients and transformed medical practices would be more likely to engage in proactive, patient-centered care and that this would result in more consistent delivery of appropriate preventive services to the right patients at the right times (see Figure 1).

\section{Methods \\ Development and Pilot Testing of the Wellness Portal}

We first assembled a Portal Advisory Committee, which included 3 clinicians, 2 office staff, and 6 patients. Based on input from 3 committee meetings, a prototype personal Wellness Portal website was developed over a 6 -month period. The prototype design incorporated management of preventive services history, tracking of personal risk factors and preferences, and generation of a tailored wellness plan. The Advisory Committee suggested a set of additional capabilities that included tracking and charting vital signs and laboratory test results, history of medical encounters, a symptom diary, management of medication lists, an immunization record for children, secure messaging with practices, and generation of an interoperable personal health record in the continuity of care record format.

We incorporated and tested these features over a 6-month period in a convenience sample of two 
practices in the Oklahoma Physicians Resource/ Research Network where elements of a preventive care delivery system had already been implemented. Survey data on satisfaction with the Portal were analyzed to describe portal utility on fourpoint Likert scales. Open-ended questions were also analyzed to describe general impressions of the Wellness Portal use. Information regarding features that facilitated or hindered the improvement of preventive care and recommendations for further Portal enhancements were compiled.

\section{Cluster Randomized Controlled Trial}

Following pilot testing, eight Oklahoma Physicians Resource/Research Network clinicians practices were selected, recruited, and enrolled into a cluster randomized controlled trial (c-RCT). Pairs of clinician practices were matched on location and practice type (urban, suburban, or rural and solo, small, or midsize) and then randomized within pairs to intervention and control arms, as proposed by Imai et al. ${ }^{8}$ Selection criteria included clinician experience with the existing patient registry (PSRS), inclusion of both young children (age $<6$ ) and adults (age $>50$ ) in the patient panel, and no previous experience with the Wellness Portal.

Two of the intervention sites were small, rural physician practices in western and central Oklahoma (each included a male physician and a female nurse practitioner), whereas the other two represented a typical, medium-size, suburban family practice environment including several male physicians, a female nurse practitioner, and a physician assistant. Correspondingly, the control group consisted of a matched sample of small and mediumsize rural and suburban practices in northeast, southwest and central Oklahoma, serving comparable patient populations in terms of demographics, socioeconomic status, and payer mix.

After practices were randomized, patients in each practice were notified about the availability of the study through handouts, flyers, and verbal communication. Interested patients were enrolled by a research assistant (RA) in the waiting room of the clinicians' offices until 70 patients from each practice were recruited $(\mathrm{N}=560)$. At the baseline visit, the RA asked patients to read and sign a patient consent form and complete baseline surveys. Patients were included in the study if they had been seen at least twice by the enrolled clinician in the last 12 months, were either children ( $\leq 6$ years older) or between 40 and 75 years old (women) or 50 and 75 years old (men), could understand and respond in English, and had a basic level of computer skills that included the ability to navigate a standard, consumer-oriented web site, use the keyboard and the mouse to interact with a browser, and understand/respond to web content phrased at 6th grade level. A sample size of approximately 400 participants (50 patients per practice) gave us $80 \%$ power to detect differences between intervention and control groups. The CONSORT diagram of the Wellness Portal trial is shown in Figure 2.

\section{Implementation Support By Practice Enbancement Assistants}

Portal implementation was facilitated by practice enhancement assistants (PEAs; trained practice facilitators) in intervention practices during the entire study period. PEAs helped practices identify study participants, map the existing preventive care workflow, tailor portal implementation in the intervention arm, recruit patients, and support portal users with basic technical assistance. Typically, portal users received a link to the portal registration page via printed study materials or over e-mail. They could sign up for the portal from home, work, or another Internetenabled location, including portal kiosks made available in the office. At the first $\log$ on, the portal would offer the following menu of options and resources: maintain demographics profile, update preventive services history, assess personal risk factors, review personalized wellness plan (recommendations), track and chart vitals and lab results, work on symptom diary, update medical encounters history, manage medication and problem list, and download a personal health record. Patients were also encouraged to print their wellness plan and discuss the plan with their doctor at their next office visit. Clinicians then worked with PEAs to implement systems in their practices to respond to preventive care recommendations (eg, improve their referral approach, incorporate health behavior counseling, and optimize coding for preventive services).

\section{Patient Surveys}

Patients were surveyed in their doctor's office via a self-administered paper questionnaire at two time 
Figure 2. CONSORT diagram of the Wellness Portal randomized controlled trial. *Participant did not complete or send the survey back to investigators and/or was unreachable. ${ }^{\dagger}$ Participant signed up as a spouse of another participant and this became evident to investigators only later.

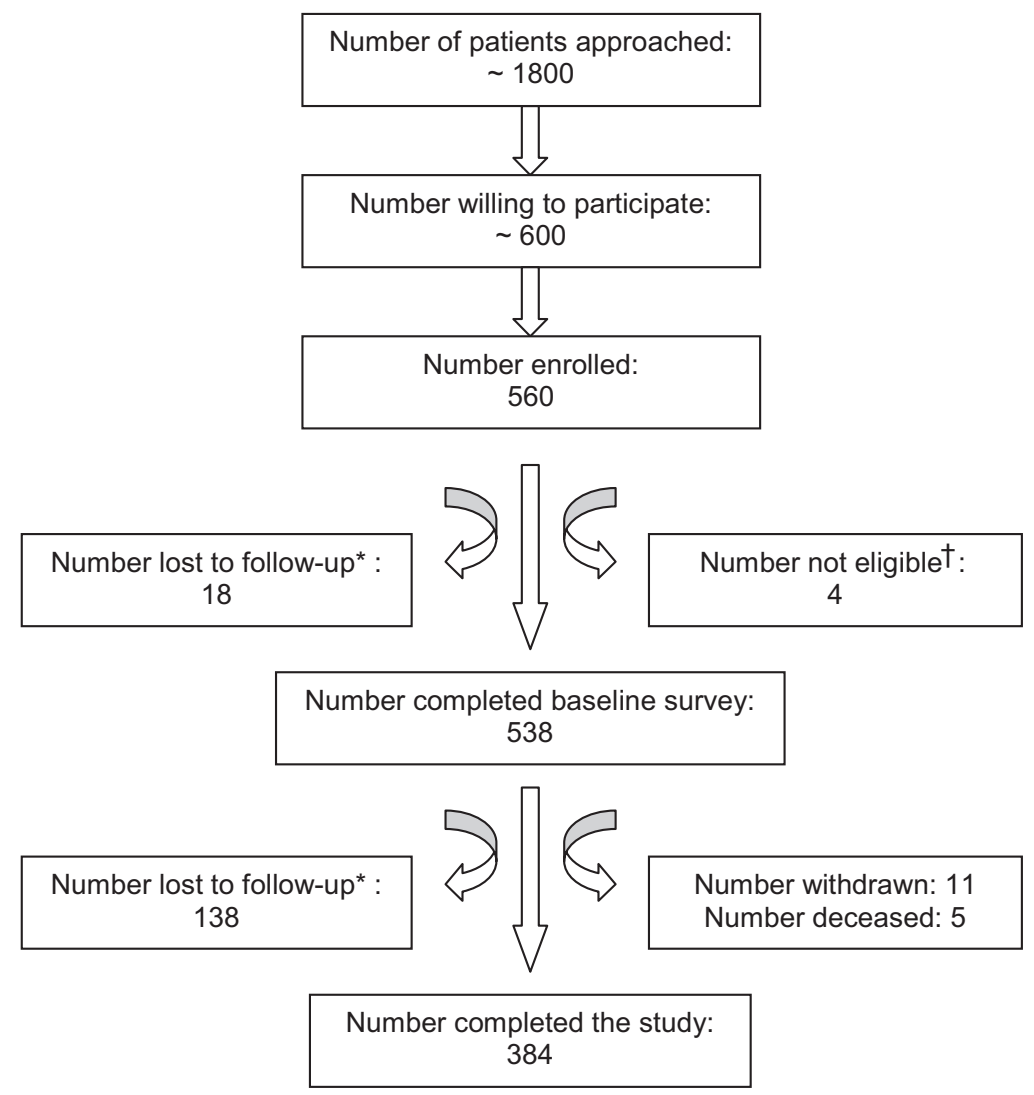

points: at baseline and 12 months later. Surveys included items describing patient experience with preventive services, satisfaction with the system of care, perception of patient-centeredness of care, patient activation, and patient empowerment. $\mathrm{Pa}$ tients in both arms of the study completed the surveys that were identical except that patients in the portal arm were asked to assess their satisfaction with the portal tool after the intervention. Patients in intervention practices were asked at enrollment to review and update their demographics, risk factor information, allergy and contraindication profile, and personal preferences through the Wellness Portal before each visit to their primary care clinician. Clinicians and their staff were asked to either use the linked practice portal (PSRS) to generate care recommendations or to ask patients to print a list of recommendations from their portal account before their next visit. Patients in control practices were not given access to the portal and they did not receive personalized recommendations or a wellness plan.

\section{Patient Experience and Patient-Centeredness of Care}

To measure patient-centeredness of care, we adapted the Consumer Assessment of Health Care Providers and Systems (CAHPS) survey developed and validated by the Agency for Health Care Research and Quality ambulatory CAHPS Initiative. We created a composite score from CAHPS survey questions 1 to 8 , plus 10 and 11 to measure the patient-centeredness of care as it relates to prevention. The 10 questions were coded as binary (yes/ no) values describing patient-clinician interactions as they relate to preventive care: assisting patients to make changes in their health habits, helping patients to make changes, encouraging patients to address health concerns, healthy diet, physical activity, worry and stress, depression, pros and cons of care options, and clinician guidance for appropriate care choices. In addition to the patient-centeredness composite score, an item measuring patient perception of provider knowledge of the patient's medical history was also included. Differ- 
ences in composite patient-centeredness scores before and after intervention were calculated for each patient and the differences were compared between control and intervention groups.

\section{Patient Activation}

Patient activation was assessed using an adapted Patient Activation Measure (PAM) questionnaire. ${ }^{10}$ The shorter form of the PAM instrument included 13 items in 3 subscales, measuring patient knowledge, confidence, and skills that demonstrate selfefficacy. ${ }^{11}$ These measures allowed us to determine the distribution of patients within the four stages of activation identified by the PAM instrument. ${ }^{11}$

\section{Patient Demographics and Health Status}

Patient age, sex, race/ethnicity, level of education, marital status, insurance coverage information, and employment status were collected via medical record reviews and baseline surveys. Patients' baseline health status was determined by documenting chronic health conditions and the number and type of office visits during a 12-month period before and during the study. We used the patients' comorbid conditions to calculate the Charlson comorbidity index ${ }^{12}$ as a measure of the severity of illness.

\section{Provision of Preventive Services}

Medical records of patients (paper and electronic) were reviewed in the practice to determine the number and type of selected preventive services received before and during the 12-month study period. We also documented the number and type of risk factors, contraindications, and personal care preferences that patients entered into the portal database. We used medical history to populate the PSRS risk engine that generated personalized preventive services recommendations based on the US Preventive Services Task Force, Advisory Committee on Immunization Practices, and American Academy of Family Physicians guidelines.

\section{Preventive Care Delivery System}

We administered an adapted version of the Assessment of Chronic Illness Care survey ${ }^{13}$ in each participating clinician's office before and after the study to evaluate the level and nature of practice system improvements, specific to the delivery of preventive services, made by clinicians and their staff. The Assessment of Chronic Illness Care addresses several chronic care model domains that include community linkages, organization of care delivery, self-management support, decision support, clinical information systems, activated patients, and prepared, proactive practice teams.

\section{Delivery of Appropriate Services At the Right Time}

We examined the delivery of preventive services under various conditions where recommendations differ based on individual patient risk factors. For example, pneumococcal vaccination is recommended for patients younger than 65 years old (2-64 years) if they have chronic cardiovascular disease, chronic pulmonary disease, or diabetes mellitus. In the absence of these chronic conditions, patients are usually eligible for pneumococcal vaccination at 65 years of age or older. Review of medical records and portal records allowed us to assess the individualization of recommendations as a result of patient input via the Wellness Portal and documentation of the patient's risk profile. We created a binary variable to describe appropriate delivery of services. If the appropriate service was delivered at the right time, the variable was assigned the value of 1 , whereas if a service should have been delivered and was not delivered, the variable was assigned the value of 0 .

\section{Patient Utilization of the Wellness Portal}

We created two variables associated with portal use during the 12-month period: (1) a dichotomous variable where the value of "use" was assigned as 1 if the patient accessed the portal and reviewed his/ her recommendations at least once; and (2) a continuous variable documenting the number of times patients have accessed the portal. Categorical variables were created to describe the type of information patients provided to clinicians through the portal that contributed to the individualization of the wellness plan. Utilization of the portal was tracked via electronic security logs that were automatically created during each user session and contained detailed information about user behavior and information exchange between users and the portal database.

\section{Analytic Strategy}

We analyzed quantitative data with the help of SAS 9.1.3 software (SAS Institute, Cary, NC). All quantitative analyses were conducted in an intention-totreat framework, adjusting for the clustering of patients within clinician practices. We conducted 
Table 1. Sex, Racial, and Ethnic Distribution of Patients at Baseline in the Randomized Controlled Trial

\begin{tabular}{lccccc}
\hline Sex & White, Non-Hispanic & Black Non-Hispanic & Hispanic & American Indian/Alaskan Native & Total, $\mathrm{n}(\%)$ \\
\hline & & & & & \\
Female & 282 & 20 & 17 & 9 & $328(61)$ \\
Male & 159 & 22 & 16 & 13 & $210(39)$ \\
Total, n (\%) & $441(82)$ & $42(8)$ & $33(6)$ & $22(4)$ & $538(100)$ \\
\hline
\end{tabular}

difference-in-differences analyses ${ }^{14}$ to determine the impact of the portal on the patient-centeredness of care. These analyses compared the magnitude of change in patient-centeredness scores between groups and time points. We also examined missed opportunities for care to gauge the impact of the portal on the delivery of preventive services. In this analysis, we compared the number of preventive services received per person between groups before and after the study using $t$ tests, considering patient clustering.

\section{Results}

\section{Pilot Study Outcomes}

The age of pilot study participants $(\mathrm{N}=30)$ ranged from 23 to 83 years (mean age, 41 years). Seventyeight percent were women, $22 \%$ were from racial or ethnic minorities, and $80 \%$ had some collegelevel education. Preliminary survey feedback indicated that participants were generally satisfied with the portal. Approximately $90 \%$ found it easy to use, $83 \%$ found it to be a valuable resource, and $80 \%$ said that it facilitated their participation in their own care. Suggestions for further improvement of the portal included shortening drop-down menus, enhancing site navigation, improving the language of on-screen instructions, and expanding options for medication list management. Lessons learned from the pilot study helped us improve the portal website, the patient recruitment approach, and participant engagement.

\section{c-RCT Outcomes}

The characteristics of study groups at baseline and those patients who did not complete the trial are listed in Tables 1 and 2. The baseline survey was completed by 422 adults 40 to 75 years of age and parents of 116 children 2 to 5 years of age $(\mathrm{N}=$ 538). At the end of the study, 279 adults and parents of 105 children $(n=384)$ completed the follow-up surveys, representing $96 \%$ of the target sample size $(\mathrm{n}$ target $=400)$. Thus, the power of the study was maintained. Medical record information was collected for all participants.

\section{Utilization and Implementation of the Wellness Portal}

During the 12-month intervention period, 280 distinct users from intervention practices logged on to the portal in 576 sessions (an average of 2 sessions

Table 2. Characteristics of Patients Enrolled in the Randomized Controlled Trial $(n=560)$ and Those Who Did Not Complete the Trial $(n=154)$ by Study Arm

\begin{tabular}{|c|c|c|c|c|}
\hline \multirow[b]{2}{*}{ Patient Characteristics } & \multicolumn{2}{|c|}{ Enrolled } & \multicolumn{2}{|c|}{ Did Not Complete } \\
\hline & Control & Intervention & Control & Intervention \\
\hline Mean age (years) & 50.5 & 54.6 & 69.0 & 60.3 \\
\hline Female sex (\%) & 59 & 63 & 48 & 51 \\
\hline Minority group (\%) & 18.5 & 18.1 & 10 & 6.1 \\
\hline High school education (\%) & $54^{*}$ & $45^{*}$ & 82 & 90 \\
\hline Average risk factors per patient (n) & 1.53 & 1.15 & 1.56 & 1.30 \\
\hline Active smokers (\%) & 15 & 17 & 25 & 19 \\
\hline Preventive service coverage at baseline (\%) & 41 & 37 & 26 & 31 \\
\hline Household income $<\$ 30,000$ per year $(\%)$ & $26^{*}$ & $40^{*}$ & $35^{*}$ & $22^{*}$ \\
\hline
\end{tabular}

${ }^{*} P<.0001$ for value pairs between control and intervention groups. 
per user). Beyond creating an account, logging in, and reviewing the website, $73 \%$ of patients used the portal in a meaningful fashion, completing at least one task (eg, entering preventive services, recording vital signs, or generating a wellness plan). Of these patients, about $12 \%$ used the portal twice, $10 \%$ used the site 3 to 5 times, and $5 \%$ were frequent users (6-25 times). One patient with chronic heart failure monitored his weight on a daily basis for a period of time (50+ user sessions). Factors associated with more frequent use included the presence of significant health conditions for which regular tracking of parameters was desirable; a higher level of interest in health, wellness, and the use of technology to improve health; and more computer/web experience.

During the course of the study, 128 patients entered 498 immunizations via the portal website, including 24 distinct vaccine doses for children (eg, 3 doses of Haemophilus influenzae type $B$ vaccine and 2 doses of measles/mumps/rubella vaccine) and adults (influenza, Pneumococcus, and adult diphtheria-tetanus). Similarly, 117 patients documented 487 preventive services, including 23 distinct service types (eg, mammography, diabetes education, smoking counseling). In addition, 77 portal users recorded 173 personal risk factors, representing 15 risk categories (eg, tobacco use, having diabetes, riding a bike). This information helped clinicians determine and personalize a list of appropriate recommendations for preventive services for individual patients.

\section{Improvements in Preventive Services Delivery}

Bivariate analyses of medical record abstraction data that accounted for clustering of patients within clinician practices indicated that adult intervention group participants received $84.4 \%$ of all recommended preventive services, whereas in the control group only $67.6 \%$ of recommended services were provided during the study period $(P<.0001)$. Similarly, adult intervention patients received $86.3 \%$ of recommended pneumococcal vaccinations, whereas control patients received only 44.6\% $(P<.0001)$. When 40- to 64-year-old patients with qualifying risk factors (eg, chronic conditions) were examined separately, $82.5 \%$ of intervention and $53.9 \%$ of control patients received a recommended pneumococcal immunization $(P<.0001)$, corresponding to a more appropriate service delivery in response to an elevated risk of infection. Overall, children in the intervention group received $95.5 \%$ of recommended immunizations compared with $87.2 \%$ in the control group $(P=.044)$. Immunization coverage for children was calculated as the number of doses received over all doses recommended at that particular age. Compared with the control group, a greater proportion of portal users had documentation of aspirin use $(78.6 \%$ of the intervention group vs $52.3 \%$ of the control group; $P<.0001$ ). These differences were significant even though patients in the intervention group had fewer visits over the 12 -month study period compared with those in the control group (average of 2.9 vs 4.3 visits; $P<$ .0001). We performed a series of 2-way analysis of variance tests to examine potential interactions between time (before and after measurements) and study groups (control and intervention). However, analyses did not indicate significant interaction between time points and group affiliation.

At baseline there was no significant difference between control and intervention groups regarding the participants' perceptions of the patient-centeredness of their care. A difference-in-differences analysis indicated that among the intervention group there was a 0.32 -point increase in the composite patient-centeredness score (calculated as a sum of 10 CAHPS survey items 1 to 8 plus 10 and 11) after the portal intervention, whereas in the control group the composite score decreased by 0.43 points $(P=.037)$.

We compared patient activation between the 2 arms of the study before and after the portal intervention using the PAM-13 questionnaire and adjusting for patient clustering. There was no difference in PAM scores at baseline between the 2 groups $(P=.44)$. Nevertheless, PAM scores (that range typically from 38.6 to 53.0$)^{11}$ indicated a modest but significant increase in patient activation in the portal intervention group compared with the control group at the end of the study (47 points vs 45 points; $P=.0014$ ). This suggests that more portal users transitioned from the second stage of activation ("confidence and knowledge to take action") to the third stage ("taking action") compared with those who did not use the portal.

\section{Missed Opportunities for Preventive Services}

At the beginning of the study, preventive service recommendations were calculated for all participants via the PSRS risk engine and based on medical history gleaned from medical record abstractions. At 
baseline, the average number of preventive service recommendations was 8.67 (SD, 3.4) per patient in the control group and 8.61 (SD, 3.2) in the intervention group (no significant difference). At the end of the trial, 11.85 (SD, 5.2) preventive services were due per patient in the control group and 9.67 (SD, 3.8 ) in the intervention group, resulting in a significant difference in the number of unaddressed preventive services per patient $\left(P<6 \times 10^{-7}\right)$.

\section{Discussion}

Results of the Wellness Portal study suggest that a comprehensive and prevention-oriented patient portal integrated into the regular process of care delivery can increase the patient-centeredness of care, improve patient activation, enhance the delivery of both age and personal risk factor -dependent preventive services, and promote the utilization of web-based personal health records.

One of the difficulties in integrating the portal into practice processes was that the portal website was available for only a small subset of patients in each clinician practice. This created logistic barriers, such as difficulty remembering which patient received the intervention and/or putting a system in place to track patient assignments for the study. When asked about the impact of the portal on patient involvement, clinicians suggested that helping patients remember to follow-up on procedures or lab work via the portal would be very helpful to trigger more participation. Several clinicians suggested that making portal use a "homework assignment" for being seen regularly (eg, via a yearly wellness visit) could also improve utilization.

Clinicians reported that the first version of the portal kiosk (a lectern-size kiosk in the waiting room) was too intimidating and conspicuous for private medical information and all supported replacing the kiosk with a small, wireless, touchscreen computer (eg, a tablet PC or an iPad). Other clinicians identified habits as the major barrier given that most patients arrived with a specific problem to be addressed and did not want to address prevention, reinforcing the necessity of dedicated, annual wellness visits that recently became reimbursable under Medicare legislation.

The Wellness Portal had a clinically significant effect on the delivery of evidence-based preventive services. Outcomes of the "missed opportunities" analyses suggested that, not surprisingly, although more preventive services were due based on the same guidelines in both patient groups as time passed, a significantly higher number of services remained unaddressed in the control group compared with the intervention group. Because the size, demographics, and clinical characteristics of the two patient groups were comparable due to randomization and clinician practice matching, it is not unreasonable to hypothesize that this difference might be explained, at least in part, by the use of the Wellness Portal that personalized care recommendations based on individual risk factors and prompted patients and clinicians to act on them. It was not possible to capture and evaluate patientclinician communication about preventive services in this study. We plan to elucidate the portaldriven shared decision-making process in future research.

Although the study showed that patients in the intervention group had fewer office visits in the course of portal implementation, our study was not designed to infer a cause-effect relationship pertaining to the impact of portal implementation on the number of patient visits that may also be affected by other factors that we did not address (eg, clinician's style of practice). We measured the socioeconomic characteristics, baseline health status, and preventive services coverage of participants that could, theoretically, predict the number of office visits, but a more definitive and targeted study should examine the connection between the implementation of a patient portal and health care utilization.

The study indicated that there was a decrease in the composite patient satisfaction and patient-centeredness of care score in the control group over time. Because the possibility of a differing effect of time on the two study groups could not be substantiated, we hypothesize that other, unmeasured factors (eg, historic factors, such as a significantly worsening economy) could have influenced general satisfaction with health care services in both groups. If this was the case, the intervention group seemed to show some improvement despite a general downward trend, further strengthening our finding.

Our study has several limitations. It was not feasible to blind study participants and PEAs who worked with practices to study group assignment; however, outcome evaluations were completed without an explicit knowledge of group affiliations. Because we relied primarily on a medical record 
abstraction approach to measure the delivery of preventive services, it was unfeasible to separate completely the effect of improved documentation from an actual increase in delivery of care. It is therefore possible that the impact of the Wellness Portal on the rate of preventive services delivery may include, in part, improved documentation. We welcome this dual effect as a typical characteristic of improving the quality of care delivery via technology and system redesign. Our previous work ${ }^{6}$ and that of others ${ }^{15}$ demonstrated a similar pattern in interventions that aimed at increasing the delivery of immunizations and other preventive services. For practical reasons, few studies were able to completely separate change in documentation from actual delivery unless a systematically utilized, reliable, alternative data source was also available as a gold standard (eg, state immunization registry). This problem of measuring the quality of care is becoming even more significant as documentation in disparate and not always connected electronic data sources compounds the complexity of individual repositories (eg, complicated structure of electronic health record databases).

In addition to the study protocol that determined the depth of portal adoption in primary care practices, limitations in use could also stem from the participation of healthier individuals who might have used the Wellness Portal less frequently simply because they had no immediate health needs or concerns. This could be a significant challenge for any technology implementation aiming at the improvement of preventive care. Thus, strategic inclusion of value-added features has been instrumental in maintaining patient interest in the portal. The Wellness Portal study also underscored the importance of alternative study designs in implementation research. These may include a practicelevel implementation phase before the study, during which technology can be integrated into the delivery of regular care as part of a systematic practice improvement effort, followed by an invitation of a subset of users to participate in a study. This approach diminishes the research artifact created by the study protocol, aligns necessary systemlevel changes with value-added resources for patients and practices, and harmonizes the two main goals of practice-based studies: improvement of the quality of care and the generation of new knowledge through research.
The portal implementation also demonstrated the importance of developing a more sophisticated understanding of patient-computer interactions and technology-related human behavior in primary care; the role of "smart design" in implementing secure, web-based resources with personal health content; patient attitudes toward preventive care; and the varying ability of practices to redesign their systems around a patient-centered technology, even when significant external support was available.

\section{References}

1. US Preventive Services Task Force. Recommendations. 2011. Available at http://www.uspreventiveservicestask force.org/recommendations.htm. Accessed March 24, 2011.

2. Yarnall KS, Pollak KI, Ostbye T, Krause KM, Michener JL. Primary care: is there enough time for prevention? Am J Public Health 2003;93:635-41.

3. Ostbye T, Yarnall KS, Krause KM, Pollak KI, Gradison M, Michener JL. Is there time for management of patients with chronic diseases in primary care? Ann Fam Med 2005;3:209-14.

4. McGlynn EA, Asch SM, Adams J, et al. The quality of health care delivered to adults in the United States. N Engl J Med 2003;348:2635-45.

5. Mangione-Smith R, DeCristofaro AH, Setodji CM, et al. The quality of ambulatory care delivered to children in the United States. N Engl J Med 2007; 357:1515-23.

6. Nagykaldi Z, Mold JW. The role of health information technology in the translation of research into practice: an Oklahoma Physicians Resource/Research Network (OKPRN) study. J Am Board Fam Med 2007;20:188-95.

7. Nagykaldi ZJ, Chou AF, Aspy CB, Mold JW. Engaging patients and clinicians through a wellness portal to improve the health of Oklahomans. J Okla State Med Assoc 2010;103:498-501.

8. Imai K, King G, Nall C. The essential role of pair matching in cluster-randomized experiments, with application to the Mexican Universal Health Insurance Evaluation. Stat Sci 2009;24:29-53.

9. Hays RD, Shaul JA, Williams VS, et al. Psychometric properties of the CAHPS 1.0 survey measures. Consumer Assessment of Health Plans Study. Med Care 1999;37:MS22-31.

10. Hibbard JH, Stockard J, Mahoney ER, Tusler M. Development of the Patient Activation Measure (PAM): conceptualizing and measuring activation in patients and consumers. Health Serv Res 2004;39: 1005-26.

11. Hibbard JH, Mahoney ER, Stockard J, Tusler M. Development and testing of a short form of the patient activation measure. Health Serv Res 2005;40:1918-30. 
12. Charlson ME, Pompei P, Ales KL, MacKenzie CR. A new method of classifying prognostic comorbidity in longitudinal studies: development and validation. J Chronic Dis 1987;40:373-83.

13. Bonomi AE, Wagner EH, Glasgow RE, VonKorff $M$. Assessment of chronic illness care (ACIC): a practical tool to measure quality improvement. Health Serv Res 2002;37:791-820.
14. Card D, Krueger AB. Minimum wages and employment: a case study of the fast-food industry in New Jersey and Pennsylvania. Am Econ Rev 1994;84: $774-5$.

15. McBride P, Underbakke G, Plane MB, et al. Improving prevention systems in primary care practices: the Health Education and Research Trial (HEART). J Fam Pract 2000;49:115-25. 